\title{
SOME FIXED POINT THEOREMS IN FUZZY METRIC SPACE
}

\author{
VYOMESH PANT
}

\begin{abstract}
The aim of the present paper is to extend the study of noncompatible maps in fuzzy metric space by using the notion of R-weak commutativity of type (Ag) in fuzzy metric space. Simultaneously, we provide an answer in fuzzy metric space, perhaps maiden, to the problem of Rhoades (page 243, [18]). We also define an analogue to the recently introduced concept of Property (E.A) by Aamri and Moutawakil [1] and then compare the results obtained by using the Property (E.A) to those obtained by using the notion of noncompatibility.
\end{abstract}

\section{Introduction}

The concept of fuzzy sets was coined by Zadeh [22] in his seminal paper in 1965. Thereafter the concept of a fuzzy metric space has been introduced and generalized in different ways by Deng [3], Erceg [5], Kaleva and Seikkala [9], Kramosil and Michalek [7], George and Veeramani [6] etc. It has also been shown that every metric induces a fuzzy metric (see Example 1 below). In 1988, Grabiec [7] extended the fixed point theorems of Banach [2] and Edelstein [4] to fuzzy metric spaces. Following Grabiec [7] and Kramosil and Michalek [8], Mishra et al. [10] obtained common fixed point theorems for compatible maps and asymptotically commuting maps on fuzzy metric space. A number of fixed point theorems have been obtained by various authors in fuzzy metric spaces by using the notion of compatibility of maps or by using its generalized or weaker forms.

In the study of fixed points of metric spaces Pant [11, 12, 14] has initiated work using the concept of noncompatible maps in metric spaces. The utility of study of noncompatible maps can be understood from the fact that while studying the common fixed point theorems of compatible maps we often require assumptions on the completeness of the space or the continuity of the mappings involved besides some contractive condition but the study of noncompatible maps can be extended to the class of nonexpansive or Lipschitz type mapping pairs even without assuming continuity of the mappings involved or completeness of the space. Recently Aamri and Moutawakil [1] introduced the property (E.A) and thus generalized the concept of noncompatible maps. The results obtained in the metric fixed point theory by using the notion of noncompatible maps or the property (E.A) are very interesting. Question arises whether, by using the concept of noncompatibility or its generalized notion, that is,

Received August 23, 2007.

2000 Mathematics Subject Classification. 47H10, 54H25.

Key words and phrases. Common fixed point, fuzzy metric space, noncompatible mappings, property (E.A), R-weak commutativity of type (Ag). 
the property (E.A), can we find equally interesting results in fuzzy metric spaces also? We answer in affirmative. In the present paper we prove common fixed point theorems for R-weakly commuting maps of type $\left(\mathrm{A}_{\mathrm{g}}\right)$ in fuzzy metric space by using the concept of noncompatibility or the property (E.A), however, without assuming either the completeness of the space or the continuity of the mappings involved. Simultaneously, we also find an answer in fuzzy metric space to the problem of Rhoades [18].

In 1994 Pant [13] introduced the concept of R-weakly commuting maps in metric spaces. Later Pathak et al. [17] generalized this concept and gave the concept of R-weakly commuting maps of type $\left(A_{g}\right)$. Vasuki [20] proved some common fixed point theorems for R-weakly commuting maps in the fuzzy metric space. Here we define the concept of R-weakly commuting maps of type $\left(\mathrm{A}_{\mathrm{g}}\right)$ and the property (E.A) in the fuzzy metric space and then prove common fixed point theorems for a pair of selfmaps.

Before we start we give some preliminaries.

Definition 1.1.([22]) Let $X$ be any set. A fuzzy set $A$ in $X$ is a function with domain $X$ and values in $[0,1]$.

Definition 1.2.([20]) A binary operation $*:[0,1] \times[0,1] \rightarrow[0,1]$ is called a continuous $t$ norm if $([0,1], *)$ is an Abelian topological monoid with the unit 1 such that $a * b \leq c * d$ whenever $a \leq c$ and $b \leq d$ for all $a, b, c, d \in[0,1]$.

Definition 1.3.([6]) The 3-tuple $(X, M, *)$ is called a fuzzy metric space if $X$ is an arbitrary set, $*$ is a continuous $t$-norm and $M$ is a fuzzy set in $X^{2} \times[0, \infty)$ satisfying the following conditions for all $x, y, z \in X$ and $t, s>0$.

(i) $M(x, y, 0)>0$

(ii) $M(x, y, t)=1$ for all $t>0$ if and only if $x=y$

(iii) $M(x, y, t)=M(y, x, t)$

(iv) $M(x, y, t) * M(y, z, s) \leq M(x, z, t+s)$

(v) $M(x, y, \cdot):[0,1) \rightarrow[0,1]$ is continuous.

Definition 1.4.([10]) Let $A$ and $B$ maps from a fuzzy metric space $(X, M, *)$ into itself. The maps $A$ and $B$ are said to be compatible (or asymptotically commuting) if, for all $t>0$,

$$
\lim _{n} M\left(A B x_{n}, B A x_{n}, t\right)=1 .
$$

Whenever $\left\{x_{n}\right\}$ is a sequence in $X$ such that $\lim _{n \rightarrow \infty} A x_{n}=\lim _{n \rightarrow \infty} B x_{n}=z$ for some $z \in X$.

From the above definition it is inferred that $A$ and $B$ are noncompatible maps from a fuzzy metric space $(X, M, *)$ into itself if $\lim _{n \rightarrow \infty} A x_{n}=\lim _{n \rightarrow \infty} B x_{n}=z$ for some $z \in X$, but either $\lim _{n} M\left(A B x_{n}, B A x_{n}, t\right) \neq 1$ or the limit does not exists.

Definition 1.5.([13]) Two maps $A$ and $S$ are called $R$-weakly commuting at a point $x$ if $d(A S x, S A x) \leq R d(A x, S x)$ for some $R>0$. A and $S$ are called pointwise $R$-weakly commuting on $X$ if given $x$ in $X$, there exists $R>0$ such that $d(A S x, S A x) \leq R d(A x, S x)$. 
Definition 1.6.([20]) Two mappings $A$ and $S$ of a fuzzy metric space $(X, M, *)$ into itself are $R$-weakly commuting provided there exists some real number $R$ such that

$M(A S x, S A x, t) \geq M(A x, S x, t / R)$ for each $x \in X$ and $t>0$.

Definition 1.7.([17]) Two selfmappings $A$ and $S$ of a metric space $(X, d)$ are called $R$-weakly commuting of type $\left(\mathrm{A}_{\mathrm{g}}\right)$ if there exists some positive real number $R$ such that

$$
d(A A x, S A x) \leq R d(A x, S x) \text { for all } x \text { in } X .
$$

Definition 1.8.([1]) Let $f$ and $g$ be two selfmappings of a metric space $(X, d)$. We say that $f$ and $g$ satisfy the property (E.A) if there exists a sequence $\left\{x_{n}\right\}$ such that

$$
\lim _{n \rightarrow \infty} f x_{n}=\lim _{n \rightarrow \infty} g x_{n}=t \text { for some } t \in X .
$$

We define the following:

Definition 1.9. Two mappings $A$ and $S$ of a fuzzy metric space $(X, M, *)$ into itself are $R$-weakly commuting of type $\left(\mathrm{A}_{\mathrm{g}}\right)$ provided there exists some real number $R$ such that

$$
M(A A x, S A x, t) \geq M(A x, S x, t / R) \text { for each } x \in X \text { and } t>0 .
$$

Definition 1.10. Let $f$ and $g$ be two selfmappings of a fuzzy metric space $(X, M, *)$. We say that $A$ and $S$ satisfy the property (E.A) if there exists a sequence $\left\{x_{n}\right\}$ such that $\lim _{n \rightarrow \infty} f x_{n}=$ $\lim _{n \rightarrow \infty} g x_{n}=t$ for some $t \in X$.

Example 1.([6]) Let $(X, d)$ be a metric space. Define $a * b=a b$ or $a * b=\min \{a, b\}$ and for all $x, y \in X, t>0$

$$
M(x, y, t)=\frac{t}{t+d(x, y)} .
$$

Then $(X, M, *)$ is a fuzzy metric space and the fuzzy metric $M$ induced by the metric $d$ is often referred to as the standard fuzzy metric.

\section{Results}

Theorem 1. Let $f$ and $g$ be pointwise R-weakly commuting selfmaps of type $\left(A_{\mathrm{g}}\right)$ of a fuzzy metric space $(X, M, *)$ such that

(i) $f X \subset g X$,

(ii) $M(f x, f y, t)>\min \{M(g x, g y, t h), M(f x, g x, t h), M(f y, g y, t h), M(f y, g x, t h), M(f x, g y, t h)\}$, $0 \leq h<1, t>0$. 
If $f$ and $g$ satisfy the property (E.A) and the range of either of $f$ or $g$ is a complete subspace of $X$, then $f$ and $g$ have a unique common fixed point.

Proof. Since $f$ and $g$ are satisfy the property (E.A), there exists a sequence $\left\{x_{n}\right\}$ in $X$ such that

(1) $\lim _{n} f x_{n}=\lim _{n} g x_{n}=p$

for some $p$ in $X$. Since $p \in f X$ and $f X \subset g X$, there exists some point $u$ in $X$ such that $p=g u$ where $p=\lim _{n} g x_{n}$. If $f u \neq g u$, the inequality

$$
\begin{aligned}
M\left(f x_{n}, f u, t\right) \geq & \min \left\{M\left(g x_{n}, g u, t h\right), M\left(f x_{n}, g x_{n}, t h\right), M(f y, g u, t h), M\left(f u, g x_{n}, t h\right),\right. \\
& \left.M\left(f x_{n}, g u, t h\right)\right\}
\end{aligned}
$$

on letting $n \rightarrow \infty$ yields

$$
\begin{aligned}
M(g u, f u, t) \geq & \min \{M(g u, g u, t h), M(g u, g u, t h), M(f u, g u, t h), M(f u, g u, t h), \\
& M(g u, g u, t h)\} \\
= & M(g u, f u, t h)
\end{aligned}
$$

Hence $f u=g u$.

Since $f$ and $g$ are R-weak commutating of type $\left(\mathrm{A}_{\mathrm{g}}\right)$, there exists $R>0$ such that

$$
M(f f u, g f u, t) \geq M(f u, g u, t / R)=1,
$$

that is, $f f u=g f u$ and $f f u=f g u=g f u=g g u$. If $f u \neq f f u$, using (ii), we get

$$
\begin{aligned}
M(f u, f f u, t)> & \min \{M(g u, g f u, t h), M(f u, g u, t h), M(f f u, g f u, t h), \\
& M(f f u, g f u, t h), M(g u, f f u, t h)\} \\
= & M(f u, f f u, t h),
\end{aligned}
$$

a contradiction. Hence, $f u=f f u$ and $f u=f f u=f g u=g f u=g g u$.

Hence $f u$ is a common fixed point of $f$ and $g$. The case when $f X$ is a complete subspace of $X$ is similar to the above case since $f X \subset g X$. Hence we have the theorem.

We now give an example to illustrate the above theorem.

Example 2. Let $X=[2,20]$ and $M$ be the usual fuzzy metric on $(X, M, *)$. Define $f, g: X \rightarrow$ $X$ as

$$
\begin{aligned}
& f x=2 \text { if } x=2 \text { or }>5, \quad f x=6 \quad \text { if } 2<x \leq 5 \\
& g 2=2, g x=x+4 \text { if } 2<x \leq 5, g x=(4 x+10) / 15 \quad \text { if } x>5
\end{aligned}
$$

also we define $M(f x, g y, t)=t /[t+d(f x, g y)]$ for all $x, y$ in $X$ and $t>0$.

Then $f$ and $g$ satisfy all the conditions of the above theorem and have a common fixed point at $x=2$. 
In this example $f X=\{2\} \cup\{6\}$ and $g X=[2,6] \cup\{7\}$. It may be seen that $f X \subset g X$. It can be verified also that $f$ and $g$ are pointwise R-weakly commuting maps of type $\left(\mathrm{A}_{\mathrm{g}}\right)$ and satisfy the (E.A) property.

Theorem 1, ibid, has been proved by using the concept of (E.A) property which has been introduced in a recent work by Aamri and Moutawakil [1]. They have shown that the (E.A) property is more general than the notion of noncompatibility. It may, however, be observed that by using the notion of noncompatible maps in place of the (E.A) property, we can not only prove the Theorem 1 above, but, in addition, we are able to show also that maps are discontinuous at their common fixed point. We do this in our next theorem and thus find out an answer in fuzzy metric space to the problem of Rhoades [18].

Theorem 2. Let $f$ and $g$ be noncompatible pointwise R-weakly commuting selfmaps of type $\left(A_{\mathrm{g}}\right)$ of a fuzzy metric space $(X, M, *)$ such that

(iii) $f X \subset g X$,

(iv) $M(f x, f y, t)>\min \{M(g x, g y, t h), M(f x, g x, t h), M(f y, g y, t h), M(f y, g x, t h), M(f x, g y, t h)\}$, $0 \leq h<1, t>0$.

If the range of $f$ or $g$ is a complete subspace of $X$, then $f$ and $g$ have a unique common fixed point and the fixed point is the point of discontinuity.

Proof. Since $f$ and $g$ are noncompatible maps, there exists a sequence $\left\{x_{n}\right\}$ in $X$ such that

(1) $\lim _{n} f x_{n}=\lim _{n} g x_{n}=p$

for some $p$ in $X$, but either $\lim _{n} M\left(f g x_{n}, g f x_{n}, t\right) \neq 1$ or the limit does not exist. Since $p \in f X$ and $f X \subset g X$, there exists some point $u$ in $X$ such that $p=g u$ where $p=\lim _{n} g x_{n}$. If $f u \neq g u$, the inequality

$$
\begin{aligned}
M\left(f x_{n}, f u, t\right)> & \min \left\{M\left(g x_{n}, g u, t h\right), M\left(f x_{n}, g x_{n}, t h\right), M(f u, g u, t h),\right. \\
& \left.M\left(f u, g x_{n}, t h\right), M\left(f x_{n}, g u, t h\right)\right\}
\end{aligned}
$$

on letting $n \rightarrow \infty$ yields

$$
M(g u, f u, t) \geq M(g u, g u, t h) .
$$

Hence $f u=g u$.

Since $f$ and $g$ are R-weak commutating of type $\left(\mathrm{A}_{\mathrm{g}}\right)$, there exists $R>0$ such that

$$
M(f f u, g f u, t) \geq M(f u, g u, t / R)=1,
$$

that is, $f u=g f u$ and $f f u=f g u=g f u=g g u$. If $f u \neq f f u$, using (iii), we get

$$
\begin{aligned}
& M(f u, f f u, t)> \min \{M(g u, g f u, t h), M(f u, g u, t h), M(f f u, g f u, t h), \\
&M(f f u, g f u, t h), M(g u, f f u, t h)\}
\end{aligned}
$$




$$
=M(f u, f f u, t h),
$$

a contradiction. Hence $f u=f f u$ and $f u=f f u=f g u=g f u=g g u$.

Hence $f u$ is a common fixed point of $f$ and $g$. The case when $f X$ is a complete subspace of $X$ is similar to the above case since $f X \subset g X$.

We now show that $f$ and $g$ are discontinuous at the common fixed point $p=f u=g u$. If possible, suppose $f$ is continuous. Then considering the sequence $\left\{x_{n}\right\}$ of (1) we get $\lim _{n} f f x_{n}=$ $f p=p$. R-weak commutativity of type $\left(\mathrm{A}_{\mathrm{g}}\right)$ implies that $M\left(f f x_{n}, g f x_{n}, t\right) \geq M\left(f x_{n}, g x_{n}, t / R\right)=$ 1 which on letting $n \rightarrow \infty$ this yields $\lim _{n} g f x_{n}=f p=p$. This, in turn, yields $\lim _{n} M\left(f g x_{n}, g f x_{n}, t\right)=$ 1. This contradicts the fact that $\lim _{n} M\left(f g x_{n}, g f x_{n}, t\right)$ is either nonzero or nonexistent for the sequence $\left\{x_{n}\right\}$ of (1). Hence $f$ is discontinuous at the fixed point. Next, suppose that $g$ is continuous. Then for the sequence $\left\{x_{n}\right\}$ of (1), we get $\lim _{n} g f x_{n}=g p=p$ and $\lim _{n} g g x_{n}=g p=$ $p$. In view of these limits, the inequality

$$
\begin{aligned}
M\left(f x_{n}, f g x_{n}, t\right) \geq & \min \left\{M\left(g x_{n}, g g x_{n}, t h\right), M\left(f x_{n}, g g x_{n}, t h\right), M\left(f g x_{n}, g g x_{n}, t h\right),\right. \\
& \left.M\left(f g x_{n}, g x_{n}, t h\right), M\left(f x_{n}, g g x_{n}, t h\right)\right\} \\
= & M\left(f x_{n}, f g x_{n}, t h\right)
\end{aligned}
$$

yields a contradiction unless $\lim _{n} f g x_{n}=f p=g p$. But $\lim _{n} f g x_{n}=g p$ and $\lim _{n} g f x_{n}=g p$ contradicts the fact that $\lim _{n} d\left(f g x_{n}, g f x_{n}\right)$ is either nonzero or nonexistent. Thus both $f$ and $g$ are discontinuous at their common fixed point. Hence we have the theorem.

We now give an example to illustrate the above theorem.

Example 3. Let $X=[2,20]$ and $M$ be the usual fuzzy metric on $(X, M, *)$. Define $f, g: X \rightarrow$ $X$ as

$$
\begin{aligned}
& f x=2 \text { if } x=2 \text { or }>5, \quad f x=6 \text { if } 2<x \leq 5 \\
& g 2=2, g x=7 \text { if } 2<x \leq 5, g x=(4 x+10) / 15 \quad \text { if } x>5
\end{aligned}
$$

also we define $M(f x, g y, t)=t /[t+d(f x, g y)]$ for all $x, y$ in $X$ and $t>0$.

Then $f$ and $g$ satisfy all the conditions of the above theorem and have a common fixed point at $x=2$.

In this example $f X=\{2\} \cup\{6\}$ and $g X=[2,6] \cup\{7\}$. It may be seen that $f X \subset g X$. It can be verified also that $f$ and $g$ are pointwise R-weakly commuting maps of type $\left(\mathrm{A}_{\mathrm{g}}\right)$. To see that $f$ and $g$ are noncompatible, let us consider a sequence $\left\{x_{n}=5+1 / n: n>1\right\}$, then $\lim _{n} f x_{n}=2$, $\lim _{n} g x_{n} \rightarrow 2, \lim _{n} f g x_{n}=6$ and $\lim _{n} g f x_{n}=2$. Hence $f$ and $g$ are noncompatible.

Remark. Aamri and Mourawakil [1] have shown that the property (E.A) introduced by them is more general then the notion of noncompatibility. It is however, worth to mention here that if we take the noncompatibility aspect instead of the property (E.A) we can show, in addition, that the mappings are discontinuous at the common fixed point. Aforesaid results illustrate our assertion in the fuzzy metric fixed point theory. This is, however, also true for the study of fixed points in metric space. 


\section{REFERENCES}

\section{References}

[1] M. Aamri and D. El Moutawakil, Some new common fixed point theorems under strict contractive conditions, J. Math. Anal. Appl. 270(2002), 181-188.

[2] S. Banach, Theorie les Operations Lineaires, Manograie Mathematyezne, Warsaw, Poland, 1932.

[3] Z. K. deng, Fuzzy pseudo-metric spaces, J. Math. Anal. Appl. 86(1982), 74-95.

[4] M. Edelstein, On fixed and periodic points under contraction mappings, J. London Math. Soc. 37(1962), 74-79.

[5] M. A. Erceg, Metric spaces in fuzzy set theory, J. Math. Anal. Appl. 69(1979), 205-230.

[6] A. George and P. Veeramani, On some results in fuzzy metric spaces, Fuzzy Sets and Systems 64(1994), 395-399.

[7] M. Grabiec, Fixed points in fuzzy metric space, Fuzzy Sets and Systems 27(1988), 385-389.

[8] O. Kramosil and J. Michalek, Fuzzy metric and statistical metric spaces, Kybernetika 11(1975), 326334.

[9] O. Kaleva and S. Seikkala, On fuzzy metric spaces, Fuzzy Sets and Systems 12(1984), 215-229.

[10] S. N. Mishra, N. Sharma and S. L. Singh, Common fixed points of maps on fuzzy metric spaces, Intenat. J. Math. Math. Sci. 17(1994), 253-258.

[11] R. P. Pant, Common fixed points of contractive maps, J. Math. Anal. Appl. 226(1998), 251-258.

[12] R. P. Pant, Noncompatible mappings and common fixed points, Soochow J. Math 26(2000), 29-35.

[13] R. P. Pant, Common fixed points of noncommuting mappings, J. Math. Anal. Appl. 188(1994), 436440.

[14] R. P. Pant, Common fixed points of Lipschitz type mapping Pairs, J. Math. Anal. Appl. 240(1999), 280-283.

[15] H. K. Pathak, Y. J. Cho and S. M. Kang, Remarks on R-weakly commuting mappings and common fixed point theorems, Bull. Korean Math. Soc. 34(1997), 247-257.

[16] Vyomesh Pant, Contractive conditions and common fixed points in fuzzy metric space, J. Fuzzy Math. 14 (2006), 267-272.

[17] Vyomesh Pant, Common fixed points in fuzzy metric space, Journal of the Indian Math. Soc. 74 (2007), 13-17.

[18] B. E. Rhoades, Contractive definitions and continuity, Contemporary Math. 72(1988), 233-245.

[19] B. M. L. Tivari and S. L. Singh, A note on recent generalizations of Jungck contraction principle, J. UPGC Soc. 3(1986), 13-18.

[20] R. Vasuki, Common fixed points for R-weakly commuting maps in fuzzy metric spaces, Indian J. Pure Appl. Math. 30(1999), 419-423.

[21] B. Schweizer and A. Sklar, Statistical metric spaces, Pacific Journal of Mathematics 10(1960), 314334.

[22] L. A. Zadeh, Fuzzy sets, Inform. and Control 8(1965), 338-353.

A-24, J. K. Puram, Choti Mukhani, Haldwani - 263 139, Nainital, Uttarakhand, India.

E-mail: vyomeshpant@yahoo.co.in 